\title{
The Beneficial Effect of Glutathione in Protection of the Central Nerves System from Damage Induced by D-galactose
}

\author{
Sadiq Safaa Mahdi ${ }^{1, *}$, Wefak Albazi ${ }^{2}$. Muna hussain Al-Aameli ${ }^{3}$ \\ 1, 2, Department of Physiology \& Pharmacology / College of Veterinary Medicine, University of Kerbala, \\ ${ }^{3}$ Department of anatomy and histology / College of Veterinary Medicine, University of Kerbala \\ *Corresponding author. Email: Alsad1234ik@gmail.com
}

\begin{abstract}
Glutathione is a tripeptide that protect cells from oxidative damage and it is also an important regulator of redox signaling, plays a role in xenobiotic detoxification, and influences cell proliferation, apoptosis, immunological function, and fibrogenesis. The aim of this study is Study the effect of antioxidant Glutathione to minimize the aging effect of dgalactose on central nerves system. And its effect on the antioxidants enzyme. Twenty-four female rat were used in the experiment. The rats divided to four group each group with six rats. And were given a daily injection of $100 \mathrm{mg} / \mathrm{kg}$ (of body weight) intraperitoneally for six weeks. Glutathione, D-Galactose, D-Galactose and Glutathione and normal saline (as same as volume used in the other group), after the end of the six week the rats were sacrifice and serum samples were taken. The study showed that use of glutathione as antioxidant reduced the constriction of beta amyloid in the body when compared to the group that was injected only with $\mathrm{D}$-galactose at level of (p-value $<0.01$ ), The main value of beta amyloid was $(12.7 \pm 3.33,26.78 \pm 4.47,8.55 \pm 1.37,22.31 \pm 1.95)$ for groups Control, d-gal GSH and GSH+D-gal respectively. , in another hand It was noted that Acetylcholinesterase has a significant increase in D- galactose group when compared to other group at level of ( $p$-value $>0.05$ ), the main value of Acetylcholinesterase was $(6.17 \pm 0.77$, $16.12 \pm 4.19,5.21 \pm 0.75,9.15 \pm 3.55$ ) for groups Control, GSH, d-gal and GSH+D-gal respectively. Glutathione peroxidase show that significant increase in D- galactose group (G3) at level of (p-value <0.01) when compared to the other group, and there is no significant change between control and GSH at level of (p-value $<0.01$ ), the main value was $(30.69 \pm 6.98,37.22 \pm 3.09,28.42 \pm 0.75,33.22 \pm 1.24)$ for groups Control, d-gal, GSH and GSH+D-gal respectively. Melanoaldihide showed that GSH group has significant decrease at level of ( $\mathrm{p}$-value $<0.05$ ) when compared to the other groups. The main value for groups was $(35.98 \pm 0.67,39.05 \pm 2.39,31.7 \pm 1.47,36.51 \pm 1.27)$ for groups Control, GSH, d-gal and GSH+D-gal respectively.
\end{abstract}

Keywords: D-galactose, Glutathione, beta amyloid, Acetylcholinesterase, aging.

\section{INTRODUCTION}

Aging changes linked to natural aging processes including shorter life, neurodegeneration, and cognitive impairments, as well as AGE production and oxidative stress, as well as transcriptional gene modifications [1, 2].

Several studies explain that aging symptom mediated by D-gal could lead to oxidative stress triggered by elevation in D-gal metabolism [3]. D-galactose, like glucose, is a monosaccharide sugar with the same chemical formula (C6H12O6). Its structure is extremely similar to that of glucose, with the exception of one hydroxyl group that is located in a different place. [4].
Galactose, on the other hand, differs from glucose in terms of chemical and metabolic properties. The most frequent dietary source of galactose is lactose, a disaccharide made composed of one molecule of glucose and one molecule of galactose [5]. D-galactose delivery to animals can cause elements of brain aging that are analogous to human brain aging in many respects. [6]. Memory loss, neuronal degeneration and apoptosis, increased oxidative stress, reduced ATP production, increased mitochondrial DNA mutation, poor mitochondrial structure, and aberrant gene expression in the brain are only some of the symptoms. [7, 8] 9]. Oxidative stress, apoptosis, and inflammation are all enhanced when an exogenous dose of D-galactose is 
given above the normal concentration, resulting in aging effects in various organs. $[9,10]$

Glutathione is a tripeptide made up of -L-glutamyl-Lcysteinylglycine that is present in all mammalian organs at concentrations ranging from 1 to $10 \mathrm{mM}$. [11]. GSH is an important regulator of redox signaling, plays a role in xenobiotic detoxification, and influences cell proliferation, apoptosis, immunological function, and fibrogenesis [12]. A difference in reactive oxygen species (ROS) generation and enzymatic and non-enzymatic antioxidants causes oxidative stress. Antioxidant biomarkers are useful for assessing the severity of disease and the antioxidants' health-promoting effects [13]

\section{MATERIALS AND METHODS}

Twenty-four (24) female white albino rat were used in this experiment and their ages between (12-14) weeks and they were (180-210) gram b.w and the animals were placed in good condition in special plastic cages and provided the animals with the appropriate conditions In terms of temperature around $\left(30 \pm 5 \mathrm{C}^{\circ}\right)$ and ventilation and The light system was $12 \mathrm{hrs}$ per day.

The animals were divided to four group each group contain 6 rats .and they were treated daily for six weeks with intraperitoneal injections of D-Galactose, GSH, DGalactose and GSH.

The first group (G1) was injected intraperitonealy with only normal slain about the same volume that use in the other groups and it was set as control group

The second group $(\mathrm{G} 2)$ was injected intraperitonealy of D-galactose (100mg/kg b.w).

The third group (G3) was injected intraperitonealy with glutathione $(100 \mathrm{mg} / \mathrm{kg}$ b.w)

The fourth group (G4) was injected with both Dgalactose and glutathione both with $(100 \mathrm{mg} / \mathrm{kg} \mathrm{b.w})$.

Blood samples were drawn after starving the animals throughout the night After Six weeks of the experiment, the animals anesthetized by chloroform Inhalation in order to control and calm the animal before the blood draw. $5 \mathrm{ml}$ blood was drawn from the heart by means of a heart puncture directly by the way the animal lay lying on its back, and sterile medical syringes of $5 \mathrm{ml}$ were used, then the blood was placed In special gel tube not containing an anticoagulant, the serum was separated by a centrifuge at a speed of $3000 \mathrm{r} / \mathrm{min}$ for 15 minutes ,the separated serum put in a Eppendorf's tubes and kept in freeze at $-20^{\circ} \mathrm{C}$ until the completion of the measurements.

\section{RESULTS AND DISCUSSION}

Results of injection of GSH and D-galactose:

The detoxification of reactive oxygen species is a crucial job in the brain, and the antioxidant glutathione plays a key role in these activities. The availability of glutathione precursors has a big impact on the glutathione concentration of brain cells. Different extracellular glutathione precursors are preferred by different kinds of brain cells [14].

D-galactose-induced brain aging occurs not only as a result of mitochondrial malfunction, but also as a result of increased oxidative stress, inflammation, and apoptosis, as well as decreased levels of brain-derived neuro trophic factors. All of these flaws eventually contribute to cognitive deterioration. Various treatment methods that target mitochondria and cognition were assessed for their efficacy in the fight against brain aging [15].

Because amyloid plaques are a hallmark of Alzheimer's disease, researchers have looked at medicines that inhibit beta-amyloid aggregation as a possible therapy. According to certain research, betaharmful amyloid's effects occur before plaques and oligomers develop as a result, scientists are exploring for strategies to prevent the harmful connections between beta-amyloid and nerve cells from occurring in the first place. [16].

Acetylcholine is one of the most prevalent neurotransmitters in the body. Cholinergic neurons employ the neurotransmitter acetylcholine as a signaling molecule. The enzyme cholinesterase plays an essential role in terminating the chemical signal between a cholinergic neuron and the nerve, muscle, or gland that is being stimulated. Acetyl cholinesterase (AChE) metabolizes acetylcholine rapidly into choline and acetic acid, making it inactive. It is necessary for the neurological system to operate normally. Acetylcholine can build when AChE is blocked, producing overstimulation of cholinergic junctions and organs regulated by cholinergic neurons [17]. The presence of acetylcholinesterase (AChE), which is often linked with -amyloid plaques18, is a typical characteristic in the Alzheimer's disease (AD) brain. A number of studies have found increased levels of MDA in the plasma/serum of people with Alzheimer's disease. [19].

In this study the result show as there is a significant deferent between groups:

Beta amyloid of D-galactose treated group (G2) showed significant $(\mathrm{p}<0.01)$ increase with compare to control (G1) and GSH (G3) after the end of the experiment as showed in Figure (1), Also, the present results showed non-significant differences $(p>0.01)$ in control (G1) and GSH (G3) groups and there is significant $(p<0.01)$ increase in $D+G(G 4)$ group when 
compared to (G1) and (G3). The main value of beta amyloid was $(12.7 \pm 3.33,26.78 \pm 4.47,8.55 \pm 1.37$, $22.31 \pm 1.95$ ) for groups Control, d-gal GSH and GSH+Dgal respectively. (LSD=8.9499). The Acetylcholinesterase of D-galactose treated group (G2) showed significant $(\mathrm{p}<0.05)$ increase with compare to other group after the end of the experiment $(16.12 \pm 4.19)$ as showed in Figure (2), Also, the present results showed non-significant differences ( $p>0.05)$ in control $(\mathrm{G} 1)$ and GSH (G3) groups and there is significant $(\mathrm{p}<0.05)$ increase in $\mathrm{D}+\mathrm{G}(\mathrm{G} 4)$ group with compare to $(\mathrm{G} 1)$ and (G3). The main value of Acetylcholinesterase was $(6.17 \pm 0.77, \quad 16.12 \pm 4.19, \quad 5.21 \pm 0.75,9.15 \pm 3.55)$ for groups Control, GSH, d-gal and GSH+D-gal respectively. ( $\mathrm{LSD}=8.2706)$. Glutathione- peroxidase (GPxs) of D-galactose treated group (G2) showed significant $(\mathrm{p}<0.01)$ increase with compare to control (G1) and GSH (G3) after the end of the experiment and there is no significant differences compeer to (G4) as showed in Figure (3), Also, the present results showed non-significant differences ( $p>0.01)$ in control $(\mathrm{G} 1)$ and GSH (G2) groups and there is significant $(\mathrm{p}<0.01)$ increase in $\mathrm{D}+\mathrm{G}(\mathrm{G} 4)$ group with compare to $(\mathrm{G} 1)$ and (G2). The main value was $(30.69 \pm 6.98,37.22 \pm 3.09$, $28.42 \pm 0.75,33.22 \pm 1.24$ ) for groups Control, d-gal, GSH and $\mathrm{GSH}+\mathrm{D}$-gal respectively $(\mathrm{LSD}=5.1336)$. Melanoaldihide of D-galactose treated group (G3) showed significant $(p<0.01)$ increase with compare to control (G1) and GSH (G3) after the end of the experiment and there is no significant differences compeer to (G4) as showed in Figure (4), Also, the present results showed non-significant differences $(\mathrm{p}>0.01)$ in control $(\mathrm{G} 1)$ and GSH $(\mathrm{G} 2)$ groups and there is significant $(p<0.01)$ increase in $D+G(G 4)$ group with compare to (G1) and (G3). The main value for groups was $(35.98 \pm 0.67,39.05 \pm 2.39,31.7 \pm 1.47,36.51 \pm 1.27)$ for groups Control, GSH, d-gal and GSH+D-gal respectively. $(\mathrm{LSD}=4.6272)$.

\section{CONCLUSION}

The study concluded that glutathione plays a crucial role in reduce the beta amyloid and reduce the risk of Alzheimer disease.

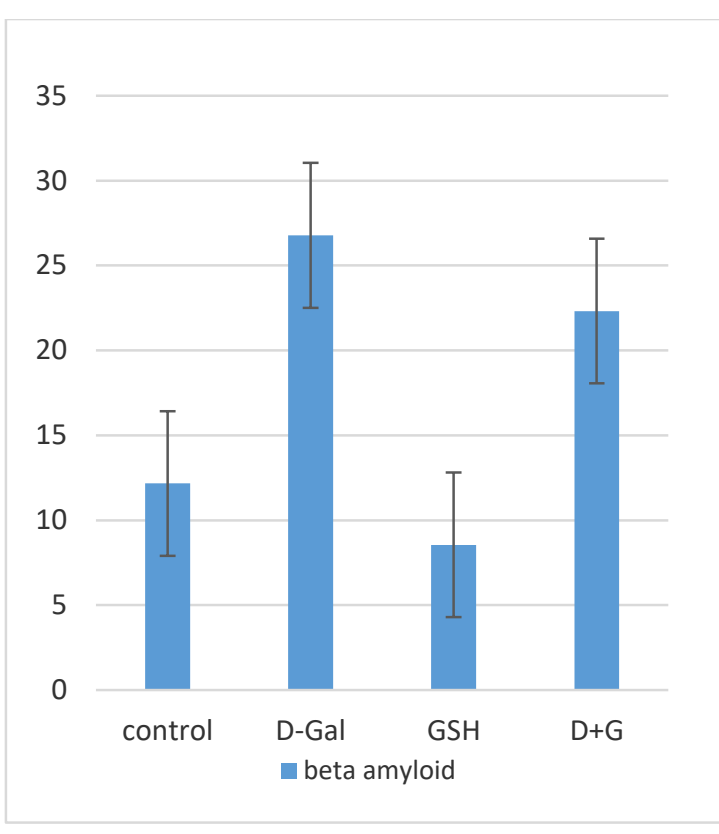

Figure 1. Effect of D-galactose, glutathione and combination at six weeks on Beta amyloid $(\mathrm{pg} / \mathrm{mL})$ in adult rats. The main value of beta amyloid was $(12.7 \pm 3.33, \quad 26.78 \pm 4.47$, $8.55 \pm 1.37,22.31 \pm 1.95$ ) for groups Control, dgal GSH and GSH+D-gal respectively.

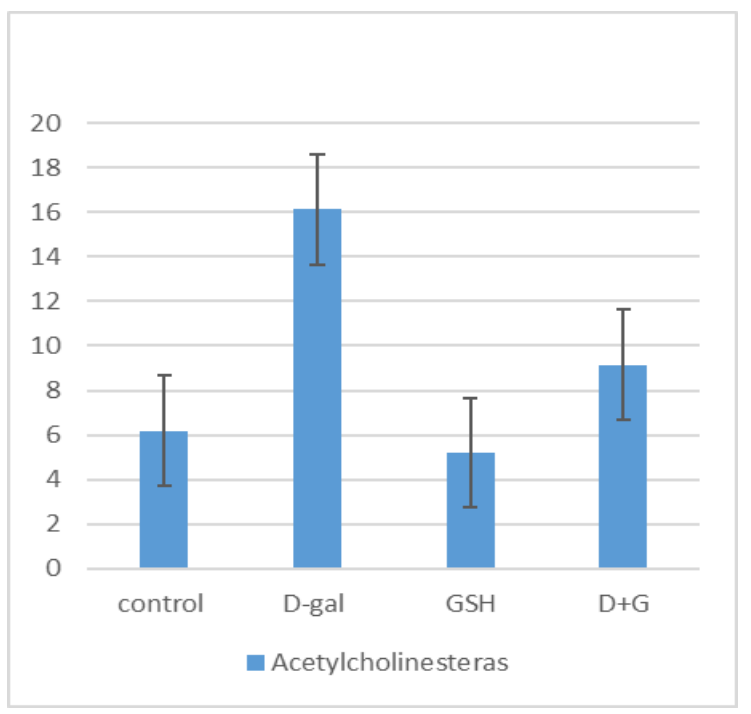

Figure 2. Effect of D-galactose, glutathione and combination at six weeks on Acetylcholinesterase $(\mathrm{pg} / \mathrm{mL})$ in adult rats. The main value of Acetylcholinesterase was $(6.17 \pm 0.77,16.12 \pm 4.19,5.21 \pm 0.75,9.15 \pm 3.5$ 5) for groups Control, GSH, d-gal and GSH+D-gal respectively. 


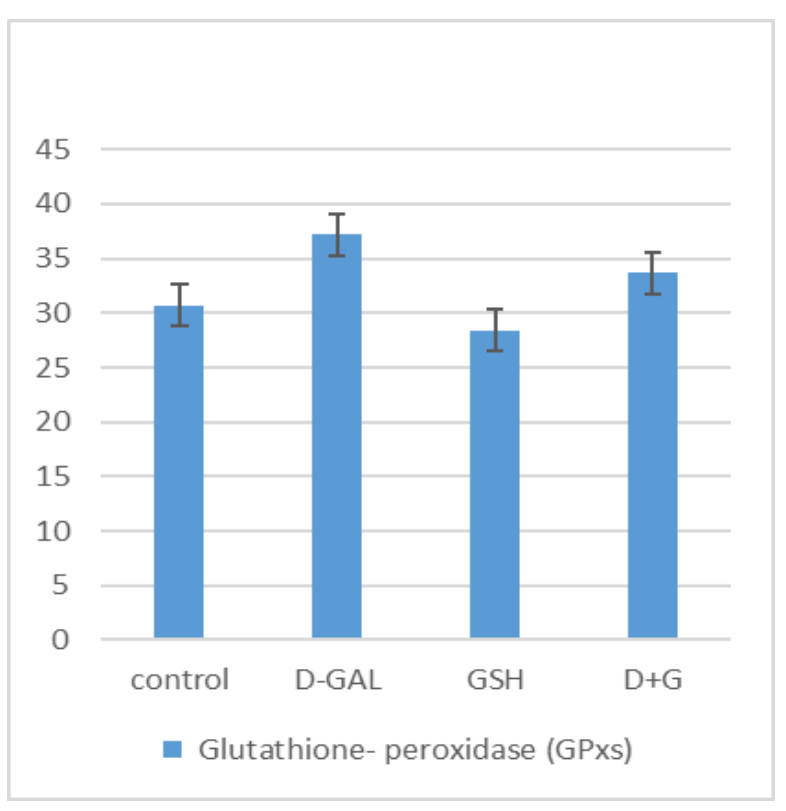

Figure 3. Effect of D-galactose, glutathione and combination at six weeks on Glutathioneperoxidase $(\mu \mathrm{g} / \mathrm{l})$ in adult rats. The main value was $\quad(30.69 \pm 6.98, \quad 37.22 \pm 3.09, \quad 28.42 \pm 0.75$, $33.22 \pm 1.24$ ) for groups Control, d-gal, GSH and $\mathrm{GSH}+\mathrm{D}$-gal respectively.

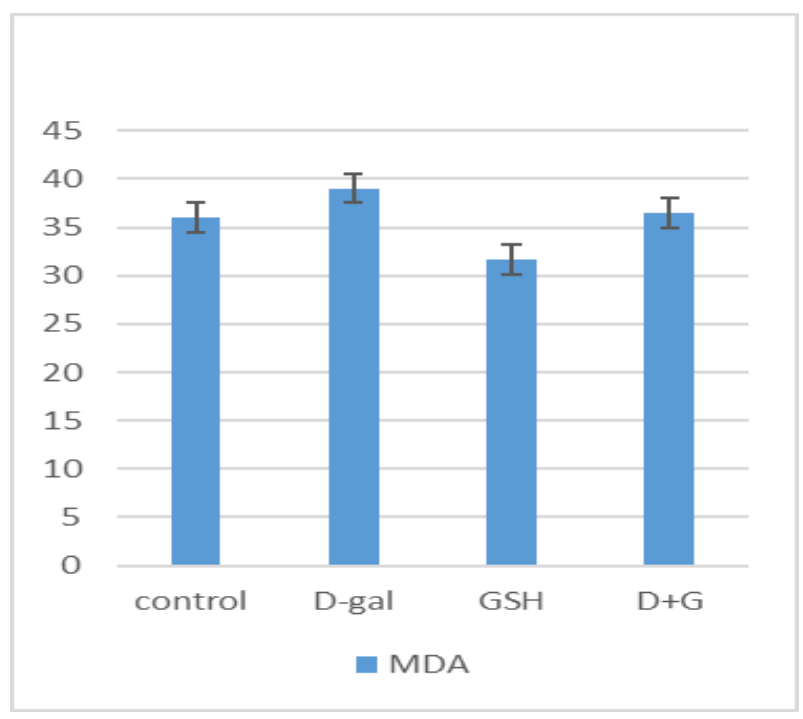

Figure 4. Effect of D-galactose, glutathione and combination at six weeks on Melanoaldihide (mol/l) in adult rats. The main value for groups was $(35.98 \pm 0.67,39.05 \pm 2.39,31.7 \pm$ $1.47,36.51 \pm 1.27$ ) for groups Control, GSH, d-gal and GSH+D-gal respectively.

\section{REFERENCES}

[1] Cui, X., Wang, L., Zuo, P., Han, Z., Fang, Z., Li, W. and Liu, J. (2004). D-galactose-caused life shortening in Drosophila melanogaster and Musca domestica is associated with oxidative stress. Biogerontology., 5(5):317-325.

[2] Tian, J., Ishibashi, K., Ishibashi, K., Reiser, K., Grebe, R., Biswal, S., Gehlbach, P. and Handa, J. T. (2005). Advanced glycation endproduct-induced aging of the retinal pigment epithelium and choroid: a comprehensive transcriptional response. Proceedings of the National Academy of Sciences., 102(33): 11846-11851.

[3] Zhou, Y.Y., Ji, X.F., Fu, J.P., Zhu, X.J., Li, R.H., Mu, C.K., Wang, C.L. and Song, W.W. (2015). Gene Transcriptional and Metabolic Profile Changes in Mimetic Aging Mice Induced by D-Galactose. PLos one., 10(7): e0132088.

[4] Rovio, S., Simolin, H., Koljonen, K., \& Sirén, H. (2008). Determination of monosaccharide composition in plant fiber materials by capillary zone electrophoresis. Journal of Chromatography A, 1185(1), 139-144.

[5] Qi, X., \& Tester, R. F. (2019). Fructose, galactose and glucose-In health and disease. Clinical nutrition ESPEN, 33, 18-28.

[6] Shwe, T., Pratchayasakul, W., Chattipakorn, N., \& Chattipakorn, S. C. (2018). Role of D-galactoseinduced brain aging and its potential used for therapeutic interventions. Experimental gerontology, 101, 13-36.

[7] Kumar, A., Prakash, A. and Dogra, S. (2011). Centella asiatica attenuates D-galactose-induced cognitive impairment, oxidative and mitochondrial dysfunction in mice. Int J Alzheimers Dis., 347569.

[8] Lei, M., Hua, X., Xiao, M., Ding, J., Han, Q. and Hu, G. (2008). Impairments of astrocytes are involved in the d-galactose-induced brain aging. Biochem Biophys Res Commun., 369:1082-1087.

[9] Ullah, F., Ali, T., Ullah, N. and Kim, M. O. (2015). Caffeine prevents d-galactose-induced cognitive deficits, oxidative stress, neuroinflammation and neurodegeneration in the adult rat brain. Neurochemistry international., 90:114-124.

[10] Qu, Z., Zhang, J., Yang, H., Huo, L., Gao, J., Chen, H. and Gao, W. (2016). Protective effect of tetrahydropalmatine against d-galactose induced memory impairment in rat. Physiology \& behavior., 154: 114-125. 
[11] Lu, S. C. (2013). Glutathione synthesis. Biochimica et Biophysica Acta (BBA)-General Subjects, 1830(5), 3143-3153.

[12] Marí, M., Morales, A., Colell, A., García-Ruiz, C., \& Fernández-Checa, J. C. (2009). Mitochondrial glutathione, a key survival antioxidant. Antioxidants $\&$ redox signaling, 11(11), 2685-2700.

[13] Marrocco, I., Altieri, F. and Peluso, I., 2017. Measurement and Clinical Significance of Biomarkers of Oxidative Stress in Humans. Oxidative Medicine and Cellular Longevity, 2017, pp.1-32.

[14] Dringen, R., 2000. Metabolism and functions of glutathione in brain. Progress in Neurobiology, 62(6), pp.649-671.

[15] Shwe, T., Pratchayasakul, W., Chattipakorn, N. and Chattipakorn, S., 2018. Role of D-galactose-induced brain aging and its potential used for therapeutic interventions. Experimental Gerontology, 101, pp.13-36.

[16] Brody, D. and Esparza, T., 2014. P4-260: AMYLOID-BETA OLIGOMERS FROM HUMAN ALZHEIMER'S DISEASE BRAIN LYSATES. Alzheimer's \& Dementia, 10, pp. 880-P881.

[17] Golomb, B., 2008. Acetylcholinesterase inhibitors and Gulf War illnesses. Proceedings of the National Academy of Sciences, 105(11), pp.4295-4300.

[18] Silveyra, M., García-Ayllón, M., de Barreda, E., Small, D., Martínez, S., Avila, J. and Sáez-Valero, J., 2012. Altered expression of brain acetylcholinesterase in FTDP-17 human tau transgenic mice. Neurobiology of Aging, 33(3), pp.624.e23-624.e34.

[19] Sinem, F., Dildar, K., Gokhan, E., Melda, B., Orhan, Y. and Filiz, M., 2010. The Serum Protein and Lipid Oxidation Marker Levels in Alzheimers Disease and Effects of Cholinesterase Inhibitors and Antipsychotic Drugs Therapy. Current Alzheimer Research, 7(5), pp.463-469. 